\title{
Corrigendum: Current drive at plasma densities required for thermonuclear reactors
}

\author{
R. Cesario, L. Amicucci, A. Cardinali, C. Castaldo, M. Marinucci, L. Panaccione, F. Santini, O. Tudisco,
} M.L. Apicella, G. Calabrò, C. Cianfarani, D. Frigione, A. Galli, G. Mazzitelli, C. Mazzotta, V. Pericoli, G. Schettini $\&$ A.A. Tuccillo and the FTU Team

Nature Communications 1:55 doi: 10.1038/ncomms1052 (2010); Published 10 Aug 2010; Updated 19 Sep 2013

In Fig. 3 of this Article, the colours of the blue and green curves were accidentally interchanged while the manuscript was being revised. In addition, the $x$ axis labels on Fig. 4 should have read 'Frequency (MHz)'. The correct versions of both figures appear below.
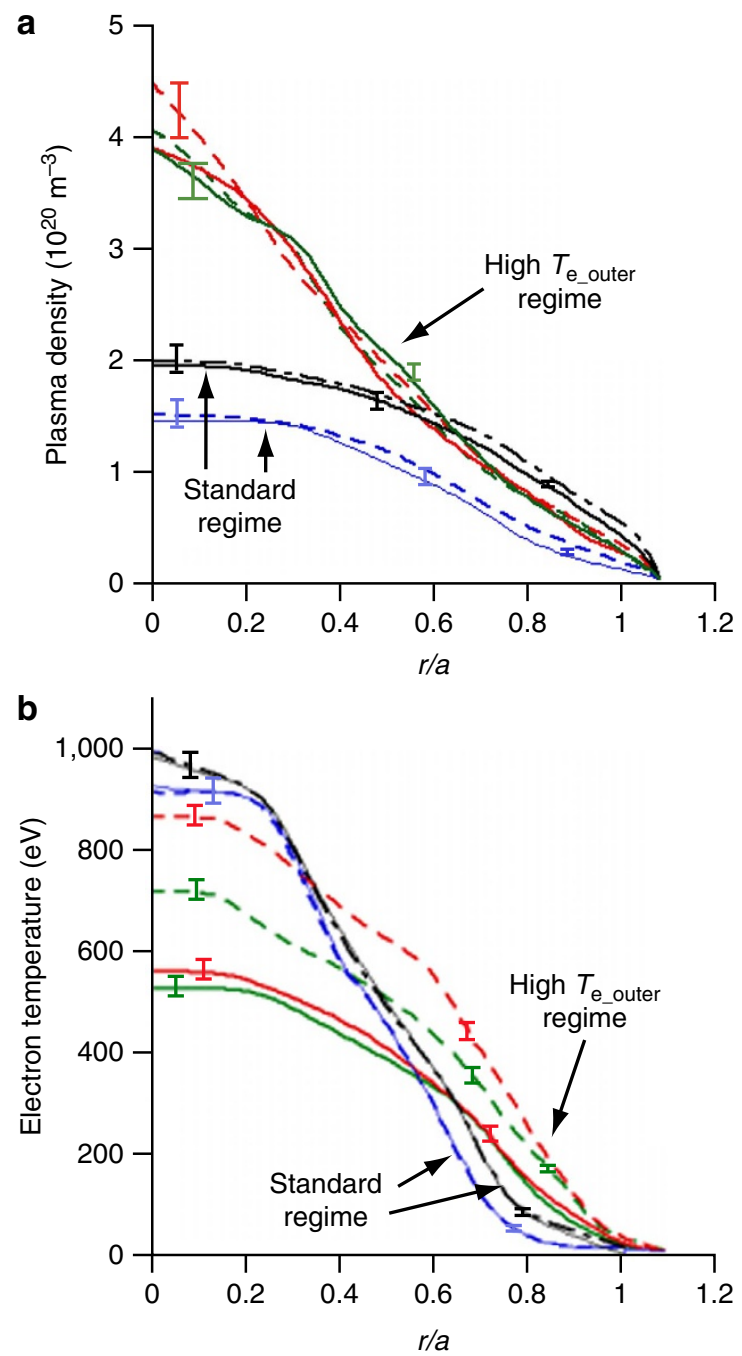

Figure 3 

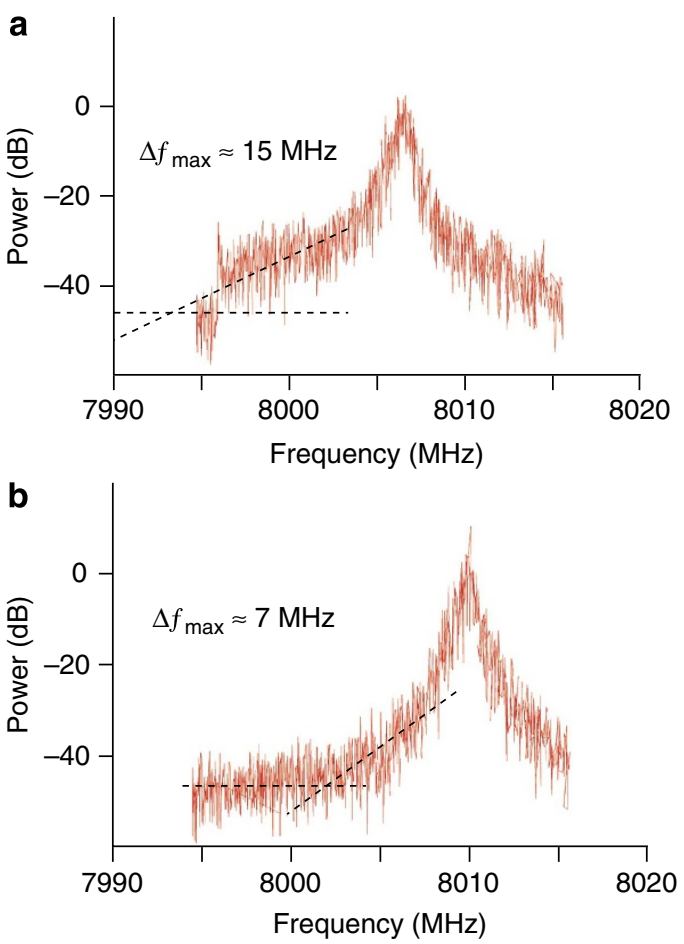

Figure 4 